\title{
Recomendaciones didácticas para adaptarse a la enseñanza remota de emergencia
}

\author{
Diana Galindo, Lorena García, Rubén García, Patricia González, Pablo C. \\ Hernández, Mireya López, Verónica Luna y Carlos I. Moreno
}

\begin{abstract}
Resumen
Con la intención de apoyar a los profesores en la transición hacia la enseñanza remota de emergencia, algunos miembros de la Red de Innovación Educativa (RIE360) hemos preparado una serie de recomendaciones relacionadas con aspectos instruccionales o didácticos en estos momentos en los que es crucial mantener una mirada innovadora en la preparación de la enseñanza debido a la pademia de covid-19. Seleccionamos aspectos puntuales de la educación en línea que han resultado clave para tener resultados favorables en esta transición hacia la educación remota de emergencia, abordamos pautas sobre planificar una clase utilizando el método de aula invertida, plantear objetivos de aprendizaje y definir estrategias dentro de un modelo de aprendizaje activo; así mismo se incluyen consideraciones para favorecer la participación de estudiantes con problemas de conectividad, el acompañamiento tutorial y se presentan sugerencias para lograr un aprendizaje -y una docencia- significativa.
\end{abstract}

Palabras clave: enseñanza remota de emergencia, contingencia educativa, planificación de la enseñanza, didáctica.

\section{INSTRUCTIONAL SUGGESTIONS FOR IMPLEMENTING EMERGENCY REMOTE TEACHING}

\begin{abstract}
With the purpose of supporting teachers in the transition towards emergency remote teaching, some members of the Network for Educational Innovation (Red de Innovación Educativa 360 or (RIE360) have prepared a series of recommendations related to instructional or didactic aspects for these moments in which it is crucial to maintain an innovative look at teaching preparation. We select specific aspects of online education that have been key for favorable results in this transition to the emergency remote education caused by the covid-19 pandemic. Instructional issues, such as establishing learning goals, planning teaching strategies, and using active learning methods at a distance are covered. Likewise, ideas on how to support students with limited access to digital devices and/or connectivity, to stimulate student engagement and participation, and to provide tutoring, all in order to achieve meaningful learning and teaching, are also covered.
\end{abstract}

Keywords: emergency remote teaching, educational contingency, course plaining, teaching.

Recepción: 17/06/2020. Aceptación: 18/08/2020. Dol: http://doi.org/10.22201/cuaieed.16076079e.2020.21.5.15 
"Recomendaciones didácticas para adaptarse a la enseñanza remota de emergencia"

\section{Diana Galindo (Universidad Anáhuac México), Lorena García (IPN), Rubén García Sánchez (UdeG), Patricia González Flores (UnAM), Pablo César Hernández Cerrito (UAM), Mireya López Acosta (Univer- sidad Anáhuac México), Verónica Luna de la Luz (UnAM), Carlos Iván Moreno Arellano (UdeG).}

La Red de Innovación Educativa (RIE360) es un grupo interinstitucional de trabajo conformado por instituciones mexicanas de educación superior, públicas y privadas. Para mayor información sobre los antecedentes de esta red, se sugiere consultar el artículo publicado en el volumen 19, número 1, enero-febrero 2018 de la RDu: http://doi.org/10.22201/codeic.16076079e.2018.v19n1.a5

\section{Datos de contacto}

Verónica Luna de la Luz: veroluluz@gmail.com

\section{Introducción}

Ante la necesidad de transitar a una modalidad de enseñanza remota de emergencia para dar respuesta a la contingencia sanitaria -que ha representado un momento que nos invita a innovar e innovarnos- es fundamental acompañar a los profesores en aquellos aspectos teórico-metodológicos necesarios de considerar ante la transición a una modalidad educativa a la que no todos están habituados (Hodges et al., 2020). Miembros de la Red de Innovación Educativa 360 (RIE360, Carvallo et al., 2018) nos hemos dado a la tarea de analizar cuáles han sido los aspectos educativos clave que han permitido tener experiencias de educación en línea exitosas en cada institución. Derivado de ello, definimos una serie de recomendaciones para los profesores con miras a orientar sus acciones antes y durante la transición a esta modalidad de enseñanza. La divulgación de estas reflexiones que emanan de la práctica de cinco universidades públicas y privadas mexicanas busca apoyar a profesores de otras instituciones educativas en el diseño de soluciones, e iniciar una discusión colectiva de los retos que enfrenta la educación superior en esta etapa.

Cabe mencionar que estas recomendaciones tienen la intención de orientar la incorporación de métodos y estrategias didácticas novedosas, que pueden ser plausibles en un contexto de enseñanza en línea, tales como el método de aula invertida o el aprendizaje activo, desde su planificación y planteamiento de objetivos. Asimismo, se incluyen consideraciones para favorecer que los estudiantes con problemas de conectividad tengan posibilidad de continuar con su formación, así como acciones para iniciar o continuar con el acompañamiento tutorial. Para finalizar, se presenta una serie de sugerencias generales orientadas a lograr una docencia significativa en esta transición. 
"Recomendaciones didácticas para adaptarse a la enseñanza remota de emergencia"

\section{Planear y estructurar una clase no presencial para aula invertida}

"Hay muchos factores involucrados en la enseñanza en línea exitosa y efectiva. Estos incluyen: el diseño del curso, actividades, contenido, la "presencia" del maestro, las interacciones entre los estudiantes y el maestro la autonomía del estudiante e incluso la administración del tiempo"

Meinecke

Un principio didáctico esencial es la planeación adecuada del proceso de enseñanza y aprendizaje. En la modalidad en línea o "virtual" toma una importancia mayúscula (Universidad Nacional de Educación a Distancia [UNED], 2020). El primer paso para que comiences a planificar es seleccionar una plataforma ya sea un LMS (Learning Management System), como D2L, Canvas, Blackboard, Moodle, u otras herramientas como Google Classroom, Zoom, Teams, etcétera. Después, necesitas organizar y estructurar las actividades de tu asignatura. Una forma ideal de estructurar tu materia virtual es establecer un calendario de sesiones y unidades (o temas) a impartir, y que hagas una diferenciación muy clara de:

- Contenidos y materiales. Selecciona materiales adecuados a tu contenido en esta nueva modalidad: presentaciones, textos, ejercicios, videos, artículos, páginas web, etcéctera.

- Actividades. Diseña las actividades que los estudiantes necesitan realizar para lograr el aprendizaje deseado: solución de ejercicios, proyectos, ensayos, lecturas comentadas, debates, infografías, videos, etcétera. Especifica las instrucciones y pasos a seguir de manera clara.

- Medio de entrega. Define el medio o herramienta a través del cual los estudiantes te enviarán las actividades, pueden ser: aula virtual, correo electrónico, nube, etcétera.

- Evaluación. Define cómo evaluarás las actividades. Por ejemplo: rúbricas, listas de cotejo, cuestionarios, etcétera.

El método de aula invertida oflipped classroom ha resultado exitoso para la enseñanza de diversas disciplinas (Strelan, Osborn y Palmer, 2020). Consiste en solicitar que los estudiantes realicen en casa las actividades que suelen llevarse a cabo en el aula (informarse sobre el contenido) y aprovechar el tiempo en clase para aquellas tareas que harían en casa (ejercicios, prácticas, proyectos orientados a la aplicación de los conocimientos). Por las condiciones actuales, se ha considerado viable su incorporación al contexto de enseñanza en línea (Berruecos-Vila, 2015). A continuación se presenta una propuesta de cómo implementarlo.

En esta nueva modalidad, lo más práctico y conveniente es reflexionar en el antes, durante y después de cada sesión o clase. En la Universidad Anáhuac México, hemos incursionado de manera decisiva en un modelo de aprendizaje 
"Recomendaciones didácticas para adaptarse a la enseñanza remota de emergencia"

invertido, teniendo una alianza con la Flipped Learning Organization. Por ello, a continuación te presentamos cómo organizar estos momentos y agregamos tips adicionales para usar esta metodología, que resulta especialmente útil para una modalidad virtual de enseñanza.

\section{Antes de la clase}

Reflexiona en la forma tradicional en la que acostumbras a impartir tus clases presenciales. Comúnmente preparamos la presentación de PowerPoint y los ejercicios que haremos durante la clase $y$, en ocasiones, les pedimos a los estudiantes que lean un capítulo o busquen algo de información antes de ésta.

Siguiendo con esta idea, para cada una de las clases en la modalidad no presencial, pregúntate y transmite muy claramente las respuestas a: ¿qué necesitan leer o revisar los alumnos?, ¿dónde les voy a colocar la información que tienen que leer?, ¿cómo voy a asegurar que han comprendido las instrucciones que deben seguir?

Alternativa con metodología de aprendizaje invertido: la palabra clave en este método es invertir, invertir lo que se hace en el antes, durante y después de las clases. Aprovecha el antes, en lugar del durante, para que los alumnos revisen los contenidos principales, prepara un video de unos 5 a 10 minutos con los aspectos importantes del tema, y solicítales que lo vean previo a la clase, para responder a algunas preguntas. Esto permitirá un mejor aprovechamiento del tiempo en el que estés (virtualmente) con tus alumnos.

\section{Durante la clase}

Pensando en una metodología "tradicional", solemos transmitir la mayor parte del contenido en el tiempo de clase, por tanto, para la clase virtual es necesario tener perfectamente preparado cómo les vas a transmitir estos contenidos a los alumnos: ¿compartirás un PowerPoint en Blackboard Collaborate, Google Meet, Zoom, Teams?, ¿cuánto tiempo expondrás?, ¿cómo vas a saber si los alumnos están atentos o tienen una duda?, ¿cuánto tiempo de clase dedicarán a hacer ejercicios (ya sea individualmente o en grupo), o discusión y qué herramienta usarás para que lo hagan?

Alternativa con metodología de aprendizaje invertido: aprovecha de manera más productiva y enriquecedora el tiempo durante la clase, dedicándolo principalmente a explicar o demostrar conceptos más complejos, ejercicios, discusiones, presentaciones, casos y resolución de dudas. Esto se puede hacer dado que la presentación de conocimientos se hizo antes de la clase.

Tip: inicia tu clase con un cuestionario sobre el contenido que previamente debieron revisar los estudiantes. 


\section{Después de la clase}

Solemos dejarles algunas tareas a los alumnos, ya sea realizar algún ejercicio o formular conclusiones de alguna lectura, entre otras. Nuevamente, a diferencia de una clase presencial en donde suelen entregarnos personalmente sus tareas, es necesario ser muy claro en la fecha, hora y a dónde te harán llegar lo que trabajaron durante la clase, ya sea por correo electrónico (no es lo más conveniente) o si lo van a subir a través de alguna herramienta seleccionada.

Alternativa con metodología de aprendizaje invertido: aprovecha el tiempo después de clase (antes de iniciar un nuevo contenido o tema), para dejar actividades muy dirigidas y útiles que te permitan evaluar los avances de tus alumnos.

En este infográfico te presentamos más consejos para organizar tus clases en los tres momentos: antes, durante y después.

\section{Pautas a considerar para preparar tu clase}

- Revisa el programa de la asignatura

- Verifica los requerimientos técnicos básicos para tu clase

- Asegúrate de contar con el acceso a una cuenta de correo electrónico

- Selecciona la herramienta de tu preferencia (Lms Blackboard, Gsuite, Teams, etcétera)

- Establece reglas de convivencia

- Revisa que cuentes con el material por clase en formato digital:

* Presentaciones

* Lecturas

* Vídeos

* Links

* Biblioteca digital

* Actividades

* Medios de evaluación (lista de cotejo, rúbrica, etcétera)

\section{Diseñar objetivos de aprendizaje realistas y actividades de aprendizaje activo para los cursos no presenciales}

Independientemente de la modalidad de tu curso, es decir, si se trata de cursos presenciales, en línea, mixtos o combinados, siempre será importante que determines con precisión lo que aprenderán los estudiantes en conjunto con los pasos y etapas para lograrlo (Eberly Center, 2020). En la coyuntura actual de la contingencia sanitaria, esto no es diferente. Si bien es probable que sea complicado mantener los mismos objetivos de aprendizaje que tenías planeados para las sesiones presenciales, sí es posible y deseable determinar objetivos de aprendizaje realistas y adecuados para la transición a cursos no presenciales.

Los objetivos de aprendizaje son enunciados que describen los conocimientos, habilidades o aptitudes que debe demostrar un estudiante al completar cada una de las lecciones que componen a un curso. Hay que considerar la importancia de que los objetivos estén articulados con los métodos 
"Recomendaciones didácticas para adaptarse a la enseñanza remota de emergencia"

de evaluación y las estrategias instruccionales (Fink, 2013). Durante el curso, el estudiante debe tener la oportunidad de demostrar lo que está aprendiendo y de recibir retroalimentación constructiva (feedback) al respecto, sea de tu parte como profesor o de sus compañeros de grupo.

Para definir objetivos de aprendizaje que puedan ser constatables, es necesario seguir algunas recomendaciones básicas: es ideal que un curso se diseñe de adelante hacia atrás (Wiggins y Tighe, 2005). Primero, se deben considerar los objetivos de aprendizaje; posteriormente, definir los modos en que se evaluaría el logro de esos aprendizajes, y, finalmente, diseñar las actividades de aprendizaje (Bowen, 2017).

La definición de los objetivos de aprendizaje debe partir de la identificación del sustantivo o lo que se busca que los estudiantes aprendan (Smith, 2012); posteriormente, se requiere establecer el nivel de profundización, es decir, necesitamos determinar qué se busca que los estudiantes sean capaces de hacer con lo que aprenden. Para ello, puede utilizarse una taxonomía como aquella propuesta originalmente por Benjamin Bloom (1956) y actualizada por Anderson y Krathwohl (2001): recordar, entender, aplicar, analizar, evaluar o crear (Churches, 2019). Finalmente, en función del nivel de profundidad, es necesario diseñar actividades de aprendizaje activo.

Las actividades pueden estar destinadas a que los estudiantes recuerden los conceptos principales; o bien, que analicen a profundidad un tema, e incluso crear algo nuevo con lo que aprenden. En cada caso, las actividades serán más elaboradas y, ¿por qué no?, diseñadas para buscar soluciones a problemas del entorno. En el manual Aprendizaje Activo, Diversidad e Inclusión (Jerez, 2014) y en el sitio de la University of California-Berkeley encontrarás varios ejemplos de actividades de aprendizaje activo, las cuales se pueden desarrollar en línea o de manera presencial. Dichas actividades podrían ser desde discusiones grupales, análisis de problemas, técnicas Think-Pair-Share para tratar rápidamente un tema, cuestionarios, encuestas en clase, creación de pósters, evaluación de tareas y trabajos entre pares, mapas conceptuales, etcétera. Lo importante es la creatividad para utilizar las técnicas que consideres más aptas para lograr que los estudiantes participen activamente en clase, profundicen en los temas y logren los objetivos.

\section{Facilitar la participación de estudiantes con problemas de conectividad}

Esta contingencia ha evidenciado que los problemas de acceso a las Tecnologías de la Información y la Comunicación (TIC) en América Latina impactan en la equidad educativa (Diálogo Interamericano, 2020), pues se ha demostrado que en la región algunos estudiantes tienen dificultades de acceso a las Tic requeridas (dispositivos y/o conectividad) para participar en actividades de aprendizaje 
"Recomendaciones didácticas para adaptarse a la enseñanza remota de emergencia" Diana Galindo, Lorena García, Rubén García, Patricia González, Pablo C. Hernández, Mireya López, Verónica Luna y Carlos I. Moreno Vol. 21, Núm. 5, septiembre-octubre 2020 Revista Digital Universitaria

en línea. Por ejemplo, en la Universidad Nacional Autónoma de México (UNAM), sabemos que $16 \%$ de los estudiantes de bachillerato no tienen una computadora en casa y que aunque el 95\% puede acceder a internet, muchos lo hacen desde su celular (Ticómetro UNAM, 2020). Encuestas recientes realizadas a los estudiantes universitarios en la UNAM indican que un porcentaje importante de ellos carece de un dispositivo, o de una conexión a internet con servicio óptimo para realizar sus actividades escolares (Román, 2020). Si las actividades que planeas demandan un acceso intensivo a un equipo propio y a conectividad con gran ancho de banda, puedes excluir a quienes carecen de ellos.

Para garantizar la equidad educativa, puedes implementar estas estrategias para posibilitar que la mayoría de tus estudiantes continúen aprendiendo y puedan acreditar tu curso:

- Distribuye a tus estudiantes la información esencial mediante una combinación de medios que incluya alguno que (i) demande poco acceso a internet, y (ii) no implique horarios específicos. Recuerda que el video es el medio que demanda más ancho de banda; mientras que el texto con imágenes fijas es el que requiere menos. Una solución muy accesible son los archivos PDF con texto y gráficos, enviados por correo o disponibles para descarga desde un aula virtual, red social, o blog. La visualización de videos y la participación de videoconferencias puede resultar una solución más simple para ti, pero puede complicar el acceso a estudiantes con poca infraestructura tecnológica.

- Establece un canal de comunicación flexible y de fácil acceso para resolver dudas de tus estudiantes. Dependiendo del número de alumnos y tu disponibilidad, puede ser el correo electrónico, una red social (Twitter o Facebook), un foro de discusión o el muro en alguna de las plataformas para cursos en línea (Moodle, Canvas, BrightSpace, Classroom).

- Considera cómo ofrecerles a todos tus estudiantes las dos funciones esenciales de un docente: la realimentación (donde brindamos información sobre la comprensión o desempeño de los alumnos como insumo para que gestionen su aprendizaje) y la mediación didáctica (en la cual les ayudamos a que comprendan contenidos difíciles, mediante ejemplos, explicaciones simplificadas, o aclaraciones de sus dudas, o promovemos el desarrollo de habilidades con recomendaciones y demostraciones de procedimientos). Puedes hacerlo enviándoles información a través de cualquiera de los medios de comunicación disponibles para interacción en línea, así como aprovechando herramientas digitales que les brinden información sobre el nivel de dominio o desempeño alcanzado, como cuestionarios o exámenes autocalificados (Wiggins, 2012).

- Ajusta tu estrategia de evaluación para que considere productos y evidencias que puedan generar incluso los alumnos que tendrán acceso limitado a un dispositivo y/o internet. Por ejemplo, un examen de libro abierto que pueda enviarse por correo electrónico y no requiera conectarse a una plataforma en un horario específico. 
- Evita o considera opcional la participación en actividades en línea síncronas, como las videoconferencias, que requieren que los estudiantes se conecten en una hora y fecha fija. Quienes tienen responsabilidades laborales o familiares, o acceso limitado a dispositivos e internet pueden tener problemas para acceder.

- Si realizas videoconferencias, graba las sesiones y compártelas con quienes no pudieron asistir. Los videos son archivos muy pesados, por lo que puedes generar un canal de YouTube, subirlos ahí y enviar el link correspondiente (si te animas, incluso puedes generar una lista de reproducción con todas las sesiones del curso).

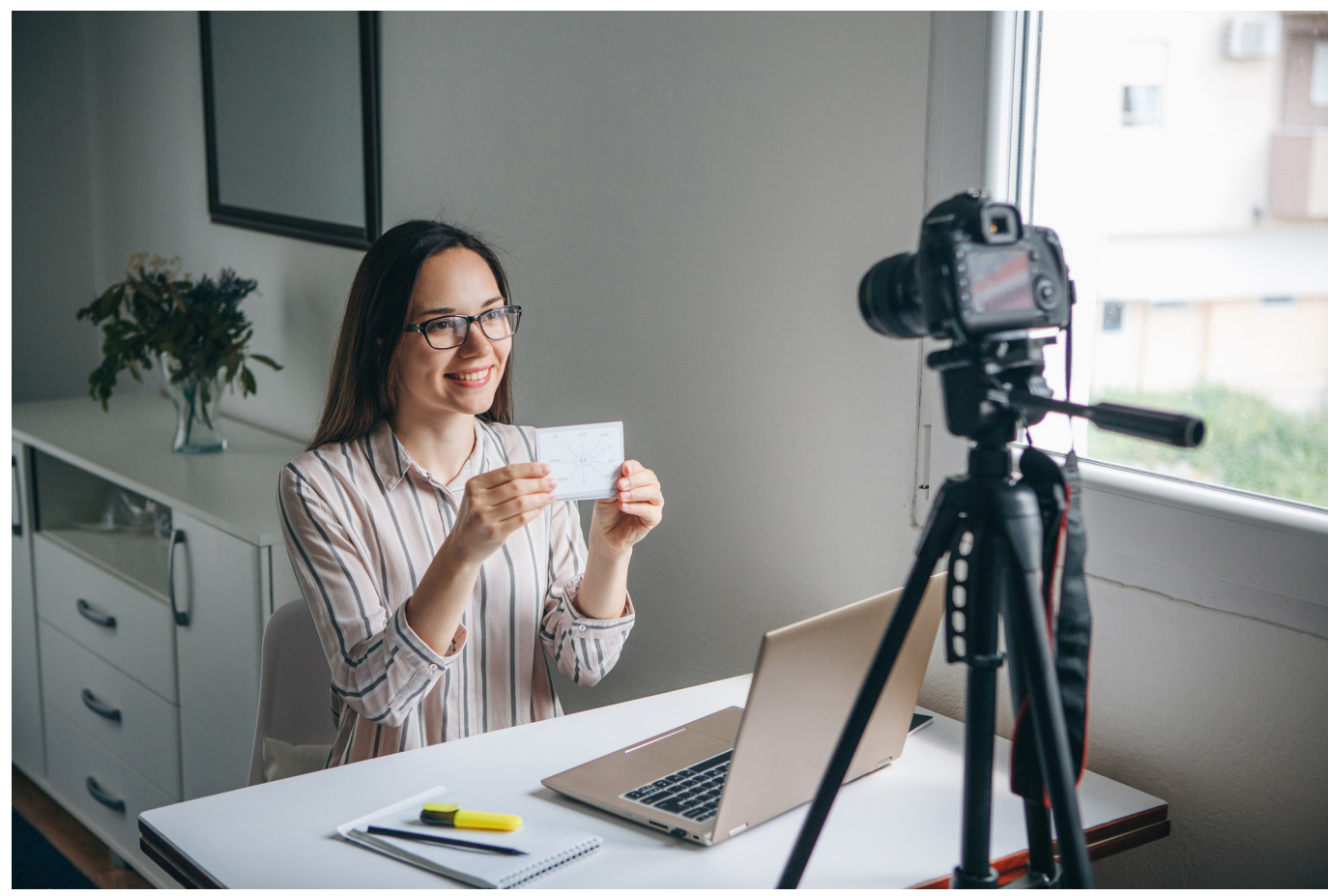

\section{Brindar acompañamiento tutorial a los estudiantes durante su estancia en casa}

Un estudio reciente en Cuba indica la importancia de continuar con el apoyo tutorial y realizar acciones precisas en tres etapas de interrelación sistémica (planificación, ejecución y control), que se realicen en un contexto de confianza a las acciones de apoyo y seguimiento en la mediación pedagógica (Díaz, Iglesias y Valdez, 2020). En esta sección se han seleccionado una serie de pautas para que continúes con esta actividad con los mejores resultados.

Durante la contingencia, es importante estar cerca de nuestros estudiantes tanto para el acompañamiento académico como para el apoyo personal que puedan necesitar. Es un hecho que los estudiantes estarán incorporando a su 
"Recomendaciones didácticas para adaptarse a la enseñanza remota de emergencia"

vida cambios radicales, entre ellos el de dar un uso académico y diferente a las tecnologías que normalmente utilizaban para crear una cultura juvenil en la que compartían música, intereses y gustos; y estarán expuestos a una tremenda cantidad de información y fake news, que les generarán inquietudes, en las que como tutores podremos auxiliar.

Para realizar un acompañamiento tutorial exitoso en esta etapa de confinamiento, toma en cuenta los siguientes puntos:

- Desarrolla la pertenencia institucional. La integración de los estudiantes con la universidad es uno de los factores que incide en su permanencia (Tinto, 1993). Idealmente comparte con tus estudiantes materiales y comunicados institucionales. Obviamente, puedes apoyarte en algunos otros que tengan gran valor académico y no estén hechos en tu institución, pero el mantener una identidad con su casa de estudios reafirmará valores y generará un sentimiento de pertenencia, lo que disminuirá la incertidumbre.

- Entiende y actúa en torno a las necesidades actuales. Dado que nuestra participación como tutores inició antes del confinamiento, es necesario realizar un nuevo diagnóstico para saber los requerimientos de los estudiantes en este momento y, con base en ellos, realizar el apoyo personal y académico. Ayudarles a organizar sus nuevas actividades y ajustar la planeación didáctica a la realidad de los jóvenes será gratificante para ellos y muy significativo para su aprendizaje.

- Mantente cerca. Los jóvenes pueden sentir ansiedad al desvincularse de su contexto académico y, por ende, de sus amigos y profesores, con quienes regularmente consultaban dudas y preocupaciones. Los noviazgos siguen, las clases continúan, se preocupan por la salud de su familia, la nueva dinámica familiar les toma por sorpresa, y un correo electrónico de nuestra parte no genera el vínculo necesario para la fluidez de la confianza. Además del trabajo en línea, una videoconferencia de diez minutos por semana en la que preguntemos ¿cómo estás?, ¿cómo te sientes en casa?, ¿cuál es el estado de salud de tu familia? pueden generar una mayor cercanía y la confianza necesaria para dar continuidad a la acción tutorial.

\section{Lograr una docencia significativa en el aprendizaje en línea}

En esta última sección se exploran los aspectos esenciales para hacer de la docencia en línea una experiencia significativa tanto para los profesores como para los estudiantes. Es una invitación que nos hace la situación actual de repensar nuestro ejercicio docente (Inter-agency Network for Education in Emergencies [INEE], 2020).

Enseñar en línea es una oportunidad para innovar los procesos de comunicación, las estrategias de aprendizaje, de motivación y de evaluación. Ante 
"Recomendaciones didácticas para adaptarse a la enseñanza remota de emergencia"

esta situación de cambio e incertidumbre se sugiere considerar los siguientes factores clave para una docencia significativa en esta modalidad (Universidad Autónoma Metropolitana [UAM], 2020):

- Diseño del curso. Como principio básico de un curso en línea, es necesario disponer al estudiante de un syllabus o plan del curso. Debe ubicarse en un lugar accesible y requiere por lo menos: una introducción, los objetivos y temas por semana, cronograma de actividades de aprendizaje, criterios de evaluación, webgrafía, así como una sección de códigos de conducta, donde se especifique el uso adecuado de citación para evitar el copy-paste y el plagio.

- Actividades de aprendizaje interactivas. Lo más motivante para un estudiante es desarrollar actividades interactivas que tengan un vínculo entre la teoría y la práctica profesional. Actividades que motiven al análisis, al pensamiento crítico, a la reflexión y a la construcción colectiva del conocimiento. En este sentido, se propone aprovechar la diversidad de recursos y actividades que ofrecen las plataformas e-learning, tales como: foros, bases de datos, glosarios, lecciones, exámenes, así como objetos de aprendizaje diseñados para resolver retos, estudios de caso, etcétera.

- Comunicación e interacción sostenida. Un elemento de motivación constante en los estudiantes es recibir realimentación oportuna. En este sentido, es preciso diferenciar entre actividades que requieren inmediata respuesta y actividades que es deseable esperar a la fecha de entrega para ofrecer realimentación. Por una parte, el mantener presencia virtual para disipar dudas y brindar seguimiento inmediato se puede realizar por medios de comunicación instantánea, como WhatsApp, Telegram u otra red social. Las actividades como tareas, foros, glosario, lecciones, pueden esperar a la fecha de entrega para brindar comentarios. Para mantener el interés en los foros discusión, es recomendable no dejar de responder en un plazo máximo a 72 horas. Es también una buena práctica que una vez a la semana exista una reunión síncrona del profesor con el grupo de estudiantes, ello se logra mediante los sistemas de videoconferencia como Zoom, Google Meet o Skype.

- Evaluación para el aprendizaje. Consideramos que la evaluación para el aprendizaje es un instrumento de mejora continua. Los medios que empleemos en la educación virtual para evaluar, tales como listas de cotejo, rúbricas, exámenes, portafolios con entregas de proyectos, ensayos, estudios de caso, webquest, entre otros, deben contribuir a subsanar las deficiencias y fortalecer los conocimientos y habilidades de los estudiantes, de acuerdo con cada nivel disciplinar. La evaluación es más que una calificación, constituye el pilar de calidad educativa. En este sentido, la evaluación tiene que brindar información al estudiante para tomar decisiones en su aprovechamiento y progreso escolar. 
"Recomendaciones didácticas para adaptarse a la enseñanza remota de emergencia"

- Factor socioemocional. El aspecto socioemocional en la educación es fundamental para integrarse en una comunidad de aprendizaje. Un estudiante que se siente satisfecho con lo aprendido, que se siente cómodo, y en un clima y ambiente de apoyo solidario, tiende a generar identidad. Ser empático ante las problemáticas y situaciones o formas de expresarse y de compartir entre las nuevas generaciones es fundamental para fortalecer la autonomía del estudiante.

Como se ha explorado en las recomendaciones, aunque estemos en una modalidad que no nos permite estar físicamente juntos, las instituciones educativas podemos contribuir de distintas maneras para que los estudiantes aprendan de manera activa y desarrollen habilidades de autorregulación de su proceso de aprendendizaje, que les permitan tomar conciencia de sus actividades, alcances, metas, capacidades y manejo del tiempo. Tenemos grandes retos para continuar con la enseñanza a distancia; con estas ideas pretendemos facilitar la misión que tenemos como profesores hacia adaptar la enseñanza a esta situación de emergencia que ha trastocado nuestra vida en todos los sentidos. Aunque estas recomendaciones no se han puesto a prueba en el contexto de esta emergencia, eventualmente se visualiza viable definir líneas de investigación que permitan dar seguimiento a su implementación.

Es importante destacar que estas recomendaciones son el resultado de un trabajo conjunto y sostenido entre las universidades de la RIE360 para apoyar al profesorado de educación superior, a partir de integrar las distintas experiencias de las instituciones que conforman la Red, así como lo ha sido la página http://rie360.mx/, en la que se han centralizado sitios que almacenan recursos educativos de acceso abierto.

\section{Referencias}

* Anderson, L. W., y Krathwohl, D. R. (2001). A Taxonomy for Learning, Teaching and Assessing: A Revision of Bloom's Taxonomy of Educational Objectives: Complete Edition. Longman.

* Berkeley Center for teaching \& Learning. (s. f.). Active Learning Strategies. Consultado el 19 de abril de 2020. https://teaching.berkeley.edu/active-learning-strategies

* Berruecos-Vila, A. M. (2015). ¿Convulsión en las aulas? El aula invertida o flipped classroom. Comunicación y pedagogía: nuevas tecnologías y recursos didácticos, 285286, 12-18.

Bloom, B. S., Engelhart, M. D., Furst, E. J., Hill, W. H. y Krathwohl, D. R. (1956). Taxonomy of educational objectives: The classification of educational goals. Handbook l: Cognitive domain. David McKay Company.

* Bowen, R. S. (2017). Understanding by Design. Vanderbilt University Center for Teaching. https://cft.vanderbilt.edu/guides-sub-pages/understanding-by-design/ 
"Recomendaciones didácticas para adaptarse a la enseñanza remota de emergencia" Diana Galindo, Lorena García, Rubén García, Patricia González, Pablo C. Hernández, Mireya López, Verónica Luna y Carlos I. Moreno Vol. 21, Núm. 5, septiembre-octubre 2020 Revista Digital Universitaria

* Carballo, A., Escamilla, J., Fueyo, E., González, P., Hernández, J., Hernández, A., López, M., Martínez, A. M. P., Montiel, M.F., Sánchez, M., Sánchez, M., Torres, R. y Velázquez, L. (2018, enero-febrero). Integración de una red de innovación educativa. La experiencia de la RIE 360. Revista Digital Universitaria (RDU, 19(1). Dol: http://doi.org/10.22201/codeic.16076079e.2018.v19n1.a5

* Churches, A. (2009). Taxonomía de Bloom para la era digital. Eduteka. http:// eduteka.icesi.edu.co/articulos/TaxonomiaBloomDigital

* Coordinación de tecnologías para la educación. (2019). tıcómetro 2019 [Resultados de la octava aplicación del cuestionario diagnóstico sobre habilidades digitales a estudiantes de primer ingreso al Bachillerato de la UNAM. Generación 2020]. DGTIC, UNAM. https://educatic.unam.mx/publicaciones/ticometro/TicometroBachillerato-2019.pdf

* Díaz, J., Iglesias, M. y Valdez, M. (2020). La tutoría a distancia: acciones del autor en la estrategia de Formación Doctoral en tiempos de covid 19. MediSur, 18(3), 478484. http://medisur.sld.cu/index.php/medisur/article/view/4678

- Eberly Center. (2020). Design \& teach a course. Carnegie Mellon University. https:// www.cmu.edu/teaching/designteach/design/learningobjectives.html

* Fink, L. D. (2013). Creating Significant Learning Experiences: An Integrated Approach to Designing College Courses, Revised and Updated. Jossey-Bass.

* Grupo de Trabajo sobre Tecnología e Innovación en Educación. (2020). La Tecnología e Innovación Educativa en el Marco de la Pandemia: Lecciones Aprendidas. Diálogo Interamericano.

* Hodges, C., Moore, S. Lockee, B. Trust, T. y Bond, A. (2020, 27 de marzo). The difference between emergency remote teaching and online learning. EDUCAUSE Review. https://er.educause.edu/articles/2020/3/the-difference-betweenemergency-remote-teaching-and-online-learning

* Inter-agency Network for Education in Emergencies (INEE). (2020, 30 de marzo). INEE response to coviD-19. https://mailchi.mp/inee.org/inee-response-to-covid-19eng?e=0170eb78b7

* Jerez, O. (2015). Aprendizaje Activo, Diversidad e Inclusión. Enfoque, Metodologías y Recomendaciones para su Implementación. Ediciones Universidad de Chille, http:// repositorio.uchile.cl/handle/2250/136742

* Román, J. (2020, 24 de agosto). En la unam, 14\% de los alumnos queda fuera de las clases en línea. La Jornada CdMx. https://www.jornada.com.mx/ultimas/ sociedad/2020/08/24/falta-o-mala-conexion-a-internet-mayor-desventaja-declases-en-linea-2918.html

* Smith, T. (2012, 2 de julio). Writing Measurable Learning Objectives. Arizona State University Teach Online. https://teachonline.asu.edu/2012/07/writingmeasurable-learning-objectives

- Tinto, V. (1993). Leaving College: Rethinking the Causes and Cures of Student Attrition (2a ed.). University of Chicago Press. 
"Recomendaciones didácticas para adaptarse a la enseñanza remota de emergencia"

Diana Galindo, Lorena García, Rubén García, Patricia González, Pablo C. Hernández, Mireya

López, Verónica Luna y Carlos I. Moreno

Vol. 21, Núm. 5, septiembre-octubre 2020

Revista Digital Universitaria

* Universidad Autónoma Metropolitana (Uam). (2020). Orientaciones didácticas para la docencia emergente de enseñanza remota. Educación virtual y a distancia en la UAM. https://www.uam.mx/educacionvirtual/uv/guias/orientaciones-didacticaspara-la-docencia-PEER.pdf

* Universidad Nacional de Educación a Distancia (Uned). (2020). Organiza y planifica tu asignatura para su desarrollo en línea. https://www.uned.es/universidad/inicio/ uned_uoc_solidaria/elearning/paso01.html

* Wiggins, G. y McTighe, J. (2005). Understanding by Design (2a ed.). Association for Supervision and Curriculum Development (ASCD).

* Wiggins, G. (2012, septiembre). Seven Keys to effective feedback. Educational Leadership, 70(1), 10-16. http://www.ascd.org/publications/educationalleadership/sept12/vol70/num01/Seven-Keys-to-Effective-Feedback.aspx

\section{Cómo CITAR ESTE ARTículo}

* Galindo, Diana, García, Lorena, García, Rubén, González, Patricia, Hernández, Pablo C., López, Mireya, Luna, Verónica y Moreno, Carlos I. (2020, septiembreoctubre). Recomendaciones didácticas para adaptarse a la enseñanza remota de emergencia. Revista Digital Universitaria (RDU), 21(5). Dol: http://doi.org/10.22201/ cuaieed.16076079e.2020.21.5.15 